\title{
Entrelacs
}

Cinéma et audiovisuel

HS | 2008

Séries télé in/out

\section{La réception des différents avatars de Betty la fea dans les espaces virtuels hispanophones, anglophones, germanophones et francophones}

Michel Magniez

\section{OpenEdition}

\section{Journals}

Édition électronique

URL : http://journals.openedition.org/entrelacs/264

DOI : $10.4000 /$ entrelacs.264

ISSN : 2261-5482

Éditeur

Éditions Téraèdre

\section{Édition imprimée}

Date de publication : 7 février 2008

ISSN : 1266-7188

Référence électronique

Michel Magniez, "La réception des différents avatars de Betty la fea dans les espaces virtuels

hispanophones, anglophones, germanophones et francophones », Entrelacs [En ligne], HS | 2008, mis en ligne le 01 août 2012, consulté le 30 avril 2019. URL : http://journals.openedition.org/entrelacs/264 ; DOI : 10.4000/entrelacs.264

Ce document a été généré automatiquement le 30 avril 2019

Tous droits réservés 


\title{
La réception des différents avatars de Betty la fea dans les espaces virtuels hispanophones, anglophones, germanophones et francophones
}

\author{
Michel Magniez
}

Selon l'écrivain et académicien Dominique Fernandez, un grand héros de roman est toujours marqué par une profonde différence, qui le sépare des autres et le place inévitablement en situation de confrontation avec son entourage, son milieu, la société en général ou avec lui-même (Le rapt de Ganymède, éd. Grasset, 1989, p.234-235). Cette réflexion littéraire peut être étendue à bon nombre de séries télévisées, dans lesquelles le héros est un être différent des autres à plus d'un titre, et bien souvent exceptionnel (que l'on pense à Hulk, Mac Gyver, Buffy, Jack Bauer ou Peter Petrelli).

Ugly Betty, ou Betty la fea dans la version hispanophone originale, est dans une situation très ambiguë par rapport à ce paradigme : à la fois proche du téléspectateur dans des situations quotidiennes, et distincte de lui dans ses aventures ou dans ses traits quasicaricaturaux, Ugly Betty est aussi éloignée d'Amanda King (Les deux font la paire) que de Sydney Bristow (Alias) et constitue, aussi bien en elle-même que dans l'histoire des séries télévisées, un cas exceptionnel. Sa différence se caractérise de manière négative (elle n'est pas belle, elle n'est pas adroite, elle n'est pas méchante, etc.) et lui donne tous les critères du parfait anti-héros. C'est son apparente banalité, et la mise en valeur de traits $a$ priori négatifs, qui ont fait son succès auprès d'un très large public.

Nous nous interrogerons sur les différents modes de réception d'une telle série, en rappelant d'abord les principales étapes du succès de l'œuvre, puis en étudiant la réception des différents avatars de la série, telle qu'elle apparaît sur les sites officiels des diffuseurs, qui tentent d'orienter et de conditionner le ressenti des téléspectateurs et internautes. Enfin, on verra que la série donne lieu à des créations nouvelles et 
personnelles sur Internet, qui prolongent le champ d'action de la série et font de la réception-même un acte de re-création.

\section{Un succès planétaire : état des lieux}

2 Diffusée pour la première fois en 1999 sur la chaîne colombienne RCN, la série Yo soy Betty la fea a connu, depuis sa création, un succès international, sous sa forme originelle comme par le biais de nombreuses adaptations locales. Aujourd'hui connue dans plus de soixantedix pays, elle a donné naissance à la Bettymania, cet engouement profond et spectaculaire d'un grand nombre de téléspectateurs de tous pays, qui s'exprime de la manière la plus forte et la plus visible sur Internet.

Le parcours atypique de cette dramedy, originaire d'Amérique du Sud, mais parvenue par exemple en France par l'intermédiaire de son adaptation allemande, met en valeur sa dimension paradoxale. A la fois internationale par son développement mondial, et universelle par l'intrigue et les sentiments qu'elle met en jeu (elle reprend, de manière revendiquée, le mythe de Cendrillon et le schème du Vilain petit canard), elle est cependant soumise également à de nombreuses adaptations locales par les chaînes et productions nationales, qui ne se contentent pas de rediffuser la version originale de la série, mais surtout la réinterprètent selon le public du pays ciblé. La série vise également à une identification individuelle du spectateur (ou de la spectatrice) à l'héroïne ou aux personnages qui l'entourent.

Cette opposition entre l'universel et l'individuel, entre le mondial et le national, se retrouve dans les différents types de réception de la série et de ses avatars. Si l'on peut observer, dans chaque pays, des constantes et des invariants comme, par exemple, la perception immédiate de la maladresse ou de la malchance de l'héroïne, le mode de réception de la série n'est cependant pas le même en fonction du pays étudié et de la version qui y est diffusée. C'est pourquoi il est nécessaire de connaître avant tout les principaux lieux de diffusion de cette série et de ses adaptations, dont voici un tableau récapitulatif rappelant le nom et les caractéristiques des différentes reprises et adaptations de la série dans les aires linguistiques envisagées :

\begin{tabular}{|l|l|l|l|l|}
\hline Pays & Titre & Actrice & Héroïne & Chaîne \\
\hline Colombie & Yo soy Betty la fea & Ana Maria Orozco & Beatriz Pinzon Solano & RCN \\
\hline Allemagne & Verliebt in Berlin & Alexandra Neldel & Lisa Plenske & Sat 1 \\
\hline USA & Ugly Betty & America G. Ferrera & Betty Suarez & ABC \\
\hline France & Le Destin de Lisa & Alexandra Neldel & Lisa Plenske & TF1 \\
\hline Mexique & La fea mas bella & Angelica Vale & Leticia Solis & Las Estrellas \\
\hline Espagne & Yo soy Betty la fea & Ana Maria Orozco & Beatriz Pinzon Solano & Antena 3 \\
\hline Espagne & Yo soy Bea & Ruth Nunez & Beatriz Perez Pizon & Tele Cinco \\
\hline
\end{tabular}


On peut d'ores et déjà ajouter que TF1 envisage de diffuser la version d'ABC à partir de la rentrée 2007.

Enfin, la série compte également de nombreux fans en dehors de ces aires, notamment en Russie, en Israël, en Inde ou aux Pays-Bas.

\section{Une réception orientée : sites des chaînes, publicité, produits dérivés}

4 Le succès de cette série n'est pas anodin, tant dans ses causes que dans ses effets. La série télévisée ne trouvant sa raison d'être que dans l'écho qu'elle aura auprès du spectateur, il est naturel que producteurs et diffuseurs de la série tentent, en amont, d'influer sur la réception individuelle et collective de leur création. Cette caractéristique vaut en particulier pour Betty la fea et ses avatars, notamment par la mise en page et le développement des sites officiels respectifs des grandes chaînes diffusant la série.

L'émission est mise en valeur sur la plupart des chaînes qui la diffusent. Sur le site de TF1, elle fait partie, avec d'autres séries et émissions de divertissement, des pages les plus populaires, bien loin devant le Journal Télévisé ou le « Programme télé », habituellement plébiscités. La chaîne Sat 1 , quant à elle, qui a connu de graves difficultés financières, a trouvé en Verliebt in Berlin un atout majeur. Il n'est donc pas étonnant que la série apparaisse d'emblée sur le site officiel de la chaîne, même si son importance commence déjà à décroître, au profit d'émissions plus anciennes que Sat 1 aimerait voir revalorisées grâce à l'attrait provoqué par Verliebt in Berlin (ces «classiques » de Sat 1 sont d'autres séries, comme Richterin Barbara Salesch, ou des émissions de divertissement comme Clever, die Show, die Wissen schafft).

La quasi-totalité des chaînes propose un ensemble de pages entièrement dédiées à Betty. Point caractéristique de la série, qui se retrouve auprès des différentes chaînes: un journal y est tenu, qui est censé être rédigé par Betty elle-même. « Hi, I’m Betty, welcome to my website » déclare par exemple Betty Suarez, avec un grand sourire, sur le site qui lui est consacré par $\mathrm{ABC}$. La fiction se dédouble alors dans le monde virtuel d'internet, à mi-chemin entre le monde imaginaire de la télévision et le monde réel du téléspectateur. « Découvre dès maintenant le nouvel album que je viens de créer » écrit Lisa sur le site de TF1. Le site allemand renonce à cet artifice de présentation, mais permet la lecture, au jour le jour, du journal intime de Hannah Refrath, protagoniste de la série, et offre l'accès à la boîte-mail de Lisa, afin de répondre à la question : "Qui écrit à notre Lisa? ». Par un effet de mise en abyme, la réception de la série devient donc à son tour objet de curiosité et donc d'une nouvelle réception par l'internaute/téléspectateur.

On notera que ces sites favorisent également la prise de parole des fans par l'intermédiaire de chats et forums, à l'exemple du site d'Antena 3 qui appelle les bettymaniacos à " parler de tout ce qui se passe dans Betty la fea ». Seule ABC propose un mode de réception très individualiste, composé principalement de videos exclusives, de photos récentes, d'information "brûlante", de podcast, et surtout de conseils pour débuter une nouvelle carrière, à l'exemple de Lisa !

Les produits dérivés sont légion, ce qui ne surprend nullement, étant donné l'ampleur du succès de la série. La palette est large, des simples fonds d'écran ou sonneries de téléphones mobiles, en passant par les livres, calendriers, films, cds, vidéos, agendas, Tshirts, colliers, et tasses, jusqu'aux plus surprenants ... sacs poubelles (!), ces derniers étant proposés par $\mathrm{ABC}$, pour «sortir ses déchets avec classe »... Les produits dérivés 
participent ainsi d'un mode de réception particulièrement lucratif, et proportionnel au succès de la série.

La publicité révèle enfin le public visé : on le suppose, dans chaque pays, majoritairement féminin et assez jeune (boissons light, vêtements féminins, produits de beauté, musique actuelle...) achetant pour le foyer ou pour soi des articles de grande consommation (lessive, nourriture, produits d'entretien...) et aimant se distraire (apéritifs, voyages, sorties, films, etc.). Il est à noter que la publicité sur les sites officiels est naturellement orientée vers un public beaucoup plus jeune (bikinis, posters, téléphones portables, téléchargements payants, etc.).

\section{L'appropriation du monde virtuel : débats et création}

5 Au-delà de ce que révèlent la publicité et les produits dérivés, il existe plusieurs modes de réception induits par la série qui permettent au spectateur d'exercer son jugement, sa liberté et sa création.

Blogs, chats et forums reprennent le déroulement et les thématiques de la série. On notera l'intérêt tout particulier porté au "phénomène Betty", et à sa portée internationale, par ceux-là même qui y participent et le propagent. Un effet boule de neige se crée, du fait de l'intérêt pour la série à l'étranger, et accroît l'intérêt du spectateur pour sa propre version nationale.

Outre des discussions sur le déroulement de l'action et l'histoire des personnages, le web donne lieu à des débats. Le questionnement sur la beauté initié par la série n'en finit pas d'être relancé: si un article des womens's news considère que Betty oblige les téléspectateurs à «se regarder dans le miroir » et à « réfléchir sur ce qu'est la véritable beauté ", les internautes et téléspectateurs restent dubitatifs, notamment quand ils découvrent la véritable apparence des actrices incarnant Betty, soudain dépossédées des caractéristiques "négatives" qui définissent leur personnage! Ces actrices "pas si moches que ça » sont alors même parfois préférées au personnage qu'elles incarnent. Une internaute déclare ainsi : "Mes enfants qui ont 17ans 14ans et moi qui en ai 37 nous suivons ts les jours les destins de lisa car nous adorons cette série et nous la suivons depuis le début. dommage que lisa n'est pas restée naturelle comme dans sa vraie vie physiquement pour la série» (sic). Position hautement paradoxale, puisque c'est justement le «handicap » de Betty qui fait l'intérêt dramatique et psychologique de la série. Mais il est évident que les chaînes elles-mêmes n'échappent pas à cette contradiction - ainsi, le générique français du Destin de Lisa est interprété par Alexandra Lucci, qui répond tant et plus aux critères traditionnels de la beauté, et dont la mise en parallèle sur le site de TF1 avec la bande-annonce de la série provoque pour l'internaute un saisissant contraste.

Comme toutes les autres séries, Ugly Betty n'échappe bien sûr pas au phénomène de partage qui caractérise l'utilisation contemporaine du net: youtube, dailymotion, et logiciels de p2p regorgent d'épisodes des différentes variantes de la série.

Enfin, par-delà les forums, blogs et chats, on relève de nombreux fanfics, fanfilms et fanarts sur le net, qui permettent aux spectateurs non seulement de s'exprimer, mais aussi de créer à leur tour des productions nouvelles en lien avec la série. Lorsqu'elles sont regroupées dans des forums, les fanfics obéissent à des règles de présentation précises (indication de la saison à laquelle il appartient, de la présence éventuelle de spoilers - c'est à dire d'éléments dévoilant la suite ou la fin de l'intrigue, etc.). Les fanarts et fanfilms sont 
régis par le droit de la propriété littéraire et artistique, mais sont largement tolérés car ils constituent un mode de diffusion de la série.

La fanfic peut prendre différentes formes: transposition (changement de point de vue), monologue intérieur d'un personnage, modification de la fin d'un épisode, insertion d'un événement, poursuite du dernier épisode, développement d'une ellipse, réécriture complète d'une péripétie, etc. Elles sont l'interface entre le monde de la série, l'étoffe des personnages, les attentes du spectateur et la liberté créatrice de l'internaute. Les auteurs sont généralement avides des commentaires des autres membres de la communauté, allant parfois jusqu'à proposer des sondages pour demander quelle fin serait la préférée des lecteurs. Les personnages secondaires y prennent souvent autant, voire plus d'importance que Betty elle-même, car ils permettent un déploiement plus large de l'imagination.

Fanart et fanfilm sont en revanche liés à l'art visuel. Le fanart est d'autant plus répandu parmi les spectateurs d'Ugly Betty que la série aborde le milieu de la mode, et permet de modifier l'apparence ou l'habillement de tel ou tel personnage. Il comprend la création de bannières et d'avatars, qui permettent l'identification de l'internaute au personnage par le biais de la photo, ou la mise en valeur d'aspects esthétiques des personnages. Le fanfilm, ou fanvid, permet de lier plusieurs arts, souvent video, musique et photo. Il consiste généralement en un patchwork d'éléments disparates (photos d'origine diverse et extraits video...) liés ensemble par un élément commun (thème, action, personnage) et/ ou un élément extérieur (musique choisie par l'internaute, commentaires, sous-titres, etc.)

6 Betty la fea est donc un exemple paradigmatique de série internationale touchant à la fois le plus grand nombre dans sa collectivité, et l'individu dans sa singularité. Les différents modes de réception permettent de distinguer certaines spécificités nationales, mais celles-ci laissent place à une création à la fois universelle et personnelle, par le biais des fanfics, fanarts et fanvids, "trouvailles artistiques », selon l'expression d'une internaute, qui font de la réception un élément constitutif à son tour d'une nouvelle étape de création.

\section{ANNEXES}

\section{Sitographie indicative commentée}

(liens valides au 01/09/2007)

1) Sites officiels des grandes chaînes francophones, germanophones, anglophones et hispanophones, classés par ordre alphabétique selon le nom de la chaîne, et suivis, le cas échant, des sites dédiés par ces chaînes à Betty et ses adaptations nationales.

ABC (USA)

http://abc.go.com

http://abc.go.com/primetime/uglybetty/index.html 


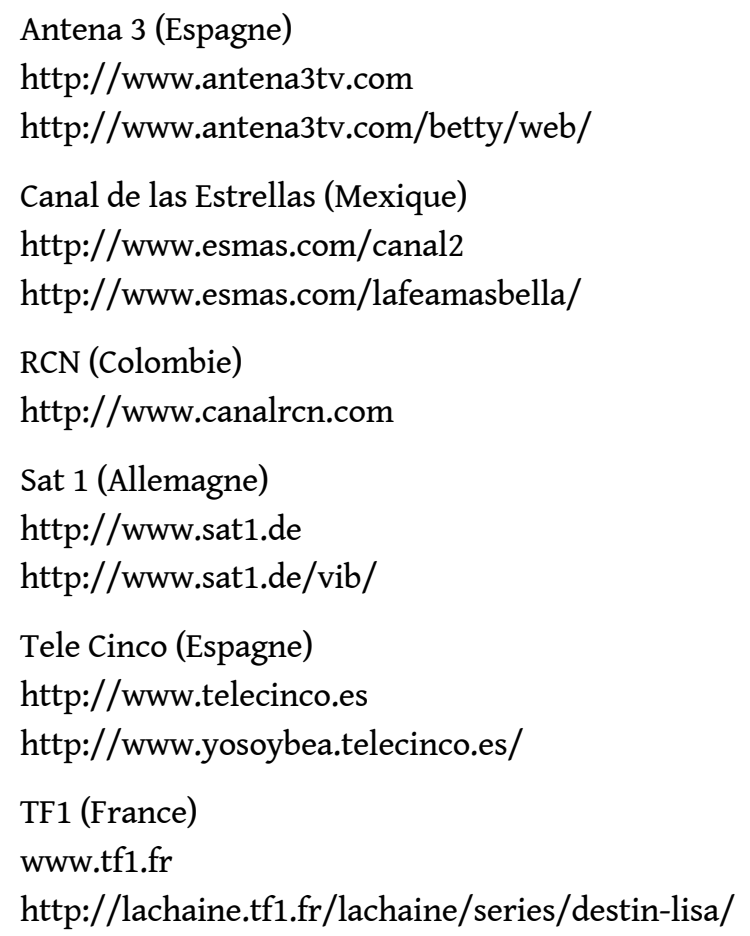

À titre de comparaison, on pourra aussi se reporter à la version néerlandaise de la série sur la chaîne Tien, dont le site spécifique est à l'adresse suivante : http://www.tien.tv/web/show/id=47920/dbid=746/typeofpage=47019

On se reportera également aux informations données par les différentes versions linguistiques de wikipedia.

\section{2) Sites, blogs et articles représentatifs}

http://www.webdelatele.com/novelas/las-versiones-de-la-telenovela-yo-soy-betty-lafea.html

Site recensant les avatars internationaux de Betty la fea

http://ledestindelisavib.leforum.eu/f11-Yo-soy-Betty-la-fea-et-les-autres-s\%C3\%A9ries-d \%C3\%A9riv\%C3\%A9es.htm

Forum qui recense les versions internationales de la série

http://makingblog.blogspot.com/2006/09/le-phnomne-betty-la-fea-continue.html Blog d'un spécialiste des médias

http://www.liberation.fr/actualite/medias/228331.FR.php

Article consacré au phénomène de la « Betty monde ».

http://elgringooo.free.fr/index.php/2007/04/04/275-alexandra-neldel-de-la-serie-ledestin-de-lisa-pas-si-moche-que-ca

Site personnel sur l'actualité des medias

http://destindelisa.serietele.info/viewforum.php $? \mathrm{f}=20$

Ensemble de fanfics sur Ugly Betty

http://www.womensenews.org/article.cfm ?aid=498

Article commentant les liens entre la série et la conception moderne de la beauté 
http://xx-pretty-alex-xx.skyblog.com/

Blog consacré à Alexandra Neldel

http://www.myvideo.de/watch/404656

Vidéo proposant « une autre fin » de Verliebt in Berlin

http://www.beepworld.de/members87/lilaloeckchen/fanfictions.htm

Ensemble de fanfictions

\section{RÉSUMÉS}

Cet article traite des différentes versions de Yo soy Betty la fea et Ugly Betty dans le monde. Il examine la façon dont ces dramedies sont perçues et utilisées par le spectateur. Le succès d'Ugly Betty est en effet inséparable des réactions qu'il a générées dans le grand public, et apparaît sur le net à travers les chats, blogs, ou forums, et particulièrement à travers les créations individuelles telles que les fanfics, fanarts et fanfilms.

\section{AUTEUR}

\section{MICHEL MAGNIEZ}

Université de Strasbourg II

Professeur agrégé au Lycée de Montdidier,

Intervenant en Classes Préparatoires aux Grandes Ecoles de Commerce 